\title{
ESTUDIOS
}

\section{Enseñanza musical en Chile: continuidades y cambios en tres reformas curriculares (1965, 1981, 1996-1998)}

\section{Music Education in Chile: Continuity and Change in three Curricular Reforms carried out in the Years 1965, 1981 and 1996-1998}

\author{
por \\ Carlos Poblete Lagos ${ }^{1}$ \\ Pontificia Universidad Católica de Chile, Chile \\ capoblel@uc.cl
}

El objeto del presente artículo es el estudio de los currícula escolares de enseñanza musical pertenecientes a tres reformas educativas chilenas (1965, 1981 y 1996-1998). Para este efecto se realiza un análisis comparativo de cada currículum, a partir de sus contenidos y propósitos fundamentales, explorando además en las relaciones que tienen dichos contenidos y propósitos con las características del contexto social y político de cada época. Para la construcción de los datos fueron utilizadas como fuentes primarias los currícula oficiales de educación musical de 1965, 1981 y 1996-1998. En forma complementaria, se recurrió a archivos documentales y literatura especializada sobre música y educación.

Palabras clave: educación musical, currículum, reformas educativas, formación de profesores de música.

The purpose of this article is the analysis of the music education curricula employed in the process of three educational reforms carried out in the years 1965, 1981 and 1996-1998. Each curriculum is analyzed and compared with the others is terms of its basic purposes and contents as relate to the features of the social and political contexts in which the educational reforms took place. The official texts of the music education curricula served as primary sources to create the data with the support of archival documents and specialized literature on music and education.

Keywords music education, curriculum, educational reforms, teacher training in music education.

${ }^{1}$ El autor agradece los comentarios y sugerencias aportados por Christian Sánchez, Alicia Poblete y Elena Guarda durante el desarrollo del presente trabajo. 


\section{INTRODUCCIÓN}

El conocimiento educativo es uno de los mayores reguladores de la estructura de la experiencia. Desde este punto de vista, uno puede preguntarse cómo formas de experiencia, identidad y relación social se evocan, se mantienen y cambian mediante la transmisión formal del conocimiento educativo y sus particularidades (Basil Bernstein, 1974) ${ }^{2}$

La génesis de la enseñanza escolar de la música tiene sus raíces en la conformación del Chile republicano, específicamente en el desarrollo de sus instituciones y políticas. Incluida desde 1847 en la formación de profesores en la naciente Escuela Normal de Preceptores de Santiago, y posteriormente ampliada a su símil femenino, la enseñanza de la música forma parte del currículo escolar en todos sus niveles a partir de 1893. Fue en ese año que -producto de la anterior llegada al país de un importante número de profesores alemanes, a instancias de los gobiernos liberales de la época- comienza a implementarse una profunda reforma al sistema educativo nacional, la cual abarca la organización de la enseñanza primaria y secundaria, la formación de profesores y la reformulación del currículo escolar de enseñanza primaria y secundaria.

Desde entonces, la enseñanza escolar de la música ${ }^{3}$ se ha mantenido dentro del currículo prescrito, adoptando diversas modalidades, en directa relación con las evoluciones de las políticas educativas y el desarrollo histórico, social y político del país.

En términos analíticos, la producción de investigaciones relacionadas con el currículo escolar provenientes del campo de la enseñanza musical se presenta principalmente en dos grandes ámbitos. En primer término, están las investigaciones que dan cuenta del "estado de la cuestión" abordadas a partir de la descripción o relato de un momento histórico particular ${ }^{4}$. En segundo término, figuran las investigaciones históricas que abordan el objeto desde una perspectiva de mayor duración estableciendo miradas comparativas entre períodos ${ }^{5}$. Asimismo, fuera del campo de la enseñanza musical existe una importante producción que da cuenta del estudio histórico del currículo escolar, sus condiciones generativas y sus relaciones, en tanto instrumento de transmisión cultural, con las instituciones, las políticas educacionales y los contextos histórico-sociales ${ }^{6}$. Sin embargo, y focalizando la búsqueda en los últimos 40 años, existe escasa producción que

\footnotetext{
2"On the Classification and Framing of the Educational Knowledge", Class, Codes and Control, vol. 3, Towards a Theory of Educational "Transmissions, Londres: Routledge and Kegan Paul, 1974. Traducción de Mario Díaz, en Revista Colombiana de Educación, $\mathrm{n}^{\circ}$ 15, 1985, p. 35.

${ }^{3}$ Hemos preferido esta denominación genérica para establecer una distinción respecto del rótulo educación musical, el cual sólo es atinente al período situado entre 1955 y 1996. Las denominaciones de gimnasia y canto, música y canto corresponden a épocas anteriores, mientras que el de artes musicales corresponde al período que va desde 1996 hasta hoy día.

${ }^{4}$ Santa Cruz 1960; Pardo y Peña 1960; Alvear 1996.

${ }^{5}$ Ottenberger 1983; Aravena et al. 1996; Sepúlveda 1996.

${ }^{6}$ Brunner 1980; Brunner et al. 1992; Cox 1987; Cox y Gysling 1987.
} 
indague aspectos específicos del currículum tales como propósitos, principios orientativos, estructura, etc., que den cuenta de las relaciones entre conocimientos y fuentes, conocimiento y condiciones generativas, conocimiento y reproducción, y finalmente, conocimiento y poder.

El foco del presente estudio radica en el abordaje de un elemento acotado y nuclear -los propósitos formativos de cada currículum- en contextos de cambios profundos en términos de política educacional, estructura curricular, principios culturales y orientaciones educativas, que establecen las condiciones genéticas en que se desarrolla la enseñanza actual de la música en las escuelas, colegios y liceos del país. Nuestro objetivo es analizar y comparar los propósitos formativos de cada currículo, dando cuenta de los principios orientativos de la asignatura entre 1965 y 1998, junto con explorar en el sentido que tiene la enseñanza de la música en cada propuesta, en el ámbito de las políticas educacionales.

Las fuentes primarias para nuestro estudio son los currícula de educación musical, elaborados a partir de la reforma de 1965 para la enseñanza básica y media, el currículum de 1981 construido para enseñanza básica y media ${ }^{7}$ y los currícula de 1996 y 1998, elaborados para la enseñanza básica y media por los proyectos MECE básica y MECE media, respectivamente ${ }^{8}$. Como fuentes secundarias hemos recurrido, además de la literatura investigativa citada anteriormente, a documentos oficiales, tesis y artículos de revistas especializadas relacionadas con el objeto de estudio. En el tratamiento de los datos se ha usado una metodología cualitativa, específicamente utilizando técnicas de análisis explicativo, estructurante y global del contenido ${ }^{9}$. Finalmente, nuestro marco teórico se apoya en los conceptos provenientes de la sociología de la educación, específicamente en el concepto de clasificación, del sociólogo británico Basil Bernstein (1988).

\section{ENSEÑANZA ESCOLAR DE LA MÚSICA EN LA REFORMA DE 1965}

\section{Antecedentes}

El período iniciado en 1965 introduce fuertes cambios en la estructura del sistema educacional, la organización del sistema escolar, la formación del profesorado y el currículum nacional. Estos cambios, enmarcados en la llamada "Revolución en libertad", impulsada por el gobierno democratacristiano del presidente Eduardo Frei Montalva (1964-1970), buscaron principalmente ampliar el acceso a la educación básica, secundaria y universitaria, en todos los niveles ${ }^{10}$. Adicionalmente, la formación de profesores es afectada, al pasar de la antigua formación de los profesores normalistas, realizada dentro del nivel secundario, a una formación especializada postsecundaria, equiparándose de esta forma con el

\footnotetext{
${ }^{7}$ Si bien existe una reforma curricular anterior, implementada en 1974, ésta no es considerada para el presente trabajo, principalmente porque los cambios introducidos afectan al ámbito de los contenidos, sin alterar la definición de los límites al interior del currículo.

${ }^{8}$ MECE: Plan de Mejoramiento de la Calidad y Equidad de la Educación.

${ }^{9}$ Flick 2004.

${ }^{10}$ Cox 1986.
} 
resto de las carreras universitarias e impactando de paso al sistema desarrollado por las escuelas normales por más de cien años. Junto a esto, la introducción de elementos de orientación psicologista de organización curriculum, ligados a los principios educativos y categorías establecidos por Benjamin Bloom y la organización de la enseñanza en un ciclo de ocho años de educación básica -antes primaria- y cuatro años de educación media -antes secundaria- dan cuenta de la magnitud de la reforma emprendida, establecida en un marco político que buscaba "la modernización de la economía y las instituciones sin postergar principios de solidaridad y el horizonte de una sociedad comunitaria” (Cox 1986: 15).

Dentro de este marco la enseñanza escolar de la música refleja los cambios en todos los niveles establecidos. En relación al currículum, se establecen nuevas reglas con respecto a los propósitos y fines, objetivos y organización de contenidos, en tanto estas nuevas reglas "apuntan contra el enciclopedismo del currículum, el verbalismo-didacticismo de la pedagogía, y la memorización como forma privilegiada de aprendizaje" (Cox 1986: 22). En este ámbito, los contenidos y habilidades presentes en el currículum de música reflejan estas orientaciones, al dar prioridad a la interpretación musical, al desarrollo de la apreciación musical y a la promoción de criterios estéticos en relación al estudio de la historia de la música, sobre un amplio repertorio que abarca principalmente la música docta, popular y folclórica ${ }^{11}$. Dichos cambios reflejan, además, el cambio de paradigma suscitado en la asignatura desde música y canto (vigente desde 1935) a educación musical, término que incluye no solo una visión integral de lo musical, sino también la orientación formativa y educadora de la asignatura.

La nueva estructura curricular divide la enseñanza básica en dos ciclos de cuatro años cada uno, organizando la enseñanza media en dos modalidades: humanista-científica (HC) y técnico-profesional (TP), cada una con una línea de formación general y otras de formación diferenciada, esta última con tres menciones para HC (Ciencias Naturales y Matemáticas, Letras y Ciencias Sociales e Históricas) y cuatro para la modalidad técnico-profesional (Industrial, Agrícola, Comercial, Servicios y técnicas especializadas). En ambas modalidades, la formación diferenciada se entrega a partir de tercer año medio, si bien ambas cuentan ya con una orientación especial desde el primer año de enseñanza media $^{12}$. En este marco, la enseñanza musical formaba parte del currículum de ambas modalidades, aunque con diferente peso horario en cada una. El siguiente cuadro resume la cantidad de horas asignadas a la asignatura por cada modalidad en cada año de enseñanza.

\footnotetext{
${ }^{11}$ Son mantenidos los conceptos utilizados originalmente en la propuesta de 1965, sin incorporar las diferentes especificaciones desarrolladas posteriormente en la discusión académica especializada, en relación al significado de cada concepto.

${ }^{12} \mathrm{Al}$ respecto, ver Revista de Educación, № 4 (nueva época), marzo 1968, p. 9.
} 


\section{CUADRO N ${ }^{\circ} 1$ \\ CANTIDAD DE HORAS ASIGNADAS A \\ EDUCACIÓN MUSICAL, $1965^{13}$}

\begin{tabular}{|c|c|c|c|c|}
\hline \multirow{2}{*}{ Modalidad } & \multirow{2}{*}{ Tipo de formación } & \multicolumn{3}{|c|}{ Cantidad de horas } \\
\hline & & Semanales & Anuales & Ciclo \\
\hline Enseñanza básica & $\begin{array}{l}\text { Dos ciclos de } 4 \text { años, } \\
\text { comunes y obligatorios } \\
\text { para todos los alumnos }\end{array}$ & 2 & 60 & 240 por cada ciclo \\
\hline \multirow{2}{*}{$\begin{array}{l}\text { Enseñanza media } \\
\text { humanista-científica }\end{array}$} & $\begin{array}{l}\text { Plan general anual } \\
\text { para } 1^{\circ} \text { y } 2^{\circ} \text { año medio }\end{array}$ & 2 & 60 & 120 para los dos años \\
\hline & $\begin{array}{l}\text { Plan diferenciado para } \\
3^{\circ} \text { y } 4^{\circ} \text { año }\end{array}$ & 2 & $60^{14}$ & 120 por 2 años \\
\hline \multirow{4}{*}{$\begin{array}{l}\text { Enseñanza media } \\
\text { técnico-profesional }\end{array}$} & $\begin{array}{l}\text { Plan General de } \\
\text { Estudios Enseñanza } \\
\text { Agrícola }\end{array}$ & - & 30 & 60 por 4 años \\
\hline & $\begin{array}{l}\text { Plan General de } \\
\text { Estudios Enseñanza } \\
\text { Industrial }\end{array}$ & - & 60 & 120 por 4 años \\
\hline & $\begin{array}{l}\text { Plan General de } \\
\text { Estudios Enseñanza } \\
\text { Comercial }\end{array}$ & - & 30 & 120 por 4 años \\
\hline & $\begin{array}{l}\text { Plan General de } \\
\text { Estudios Servicios y } \\
\text { técnicas especializadas }\end{array}$ & - & 60 & 120 por 4 años \\
\hline
\end{tabular}

\subsection{Currículum de educación musical 1965}

La reforma de 1965 considera la enseñanza musical como parte de las asignaturas constitutivas de la enseñanza básica y del plan general de la enseñanza media. En este marco, la enseñanza musical era obligatoria para el primer y segundo ciclo de la enseñanza básica. En la enseñanza media, en tanto, la obligatoriedad de la asignatura va a depender de la modalidad de enseñanza. En el plan general HC, la enseñanza musical es obligatoria en el plan general y optativa para la formación diferenciada (debiéndose escoger una asignatura de Artes, con 120 horas asignadas para dos años). En la modalidad técnico-profesional, su presencia varía tanto en la

\footnotetext{
${ }^{13}$ Cabe mencionar que para la educación técnico-profesional son detalladas en las fuentes solo las horas anuales, sin especificar la equivalencia en horas semanales.

${ }^{14}$ Se desglosan de las 120 horas consignadas para los dos años, los cuales tienen carácter electivo con Artes Plásticas.
} 
obligatoriedad como en la organización interna de las horas asignadas de acuerdo a las características del tipo de formación al interior de la modalidad ${ }^{15}$.

Internamente, el currículum de educación musical de 1965 se organiza en función de áreas de contenidos que clasifican temáticamente el conocimiento musical, los cuales cambian de acuerdo al ciclo de enseñanza en que se encuentren. Para el primer ciclo (entre el primer y cuarto año de enseñanza básica), los contenidos se organizan principalmente en torno a la práctica musical por medio de la ejecución de rondas, canciones, melodías tradicionales y del folclore, junto al desarrollo de la conciencia corporal por medio de la integración del cuerpo en actividades de interpretación vocal e instrumental, apreciación musical y la creación, un conjunto de ámbitos que se desarrollan en los ciclos posteriores. En el segundo ciclo, los ámbitos de contenidos se expanden a nueve, presentando orientaciones tanto de orden cognitivo como procedimental. En tal sentido figuran los elementos musicales básicos de la música; los fenómenos (sonoros) naturales audibles; la clasificación de voces humanas e instrumentos en general; la clasificación de conjuntos instrumentales por familias de instrumentos; la ejecución de canciones, melodías y danzas tradicionales nacionales, latinoamericanas y universales; la audición de obras musicales de fácil comprensión, chilenas, americanas y universales; la improvisación y creación de motivos musicales simples; la construcción de instrumentos musicales con materiales del medio; las profesiones relacionadas con la música.

Para el plan general de la enseñanza media, los contenidos se organizan en cuatro ejes, a saber, lectura musical, creación, audición dirigida, práctica de música vocal e instrumental para solistas y conjuntos. Este ciclo se caracteriza por su orientación hacia la práctica musical relativa tanto a la ejecución como también al desarrollo de la discriminación auditiva, el conocimiento histórico de estilos, obras y autores, así como algunos lineamientos relativos a la formación estética de auditores. Por su parte, el plan diferenciado continúa con los ejes de lectura y audición dirigida, suprimiendo en tanto eje la práctica de repertorio vocal e instrumental, y ampliándose a un nuevo eje (armonía).

Resulta interesante la inclusión dentro del programa de un repertorio variado en cuanto a estilo y procedencia. Si bien la matriz teórica y de análisis musical se encuentra siempre apegada a la tradición de la música docta, el incluir repertorio folclórico y popular (tanto nacional como extranjero), así como un panorama histórico-estilístico desde la antigüedad hasta el romanticismo en tercer año, y música del siglo XX durante el cuarto año, es elocuente acerca del pluralismo con que se concebía la formación musical para los establecimientos de la república, así como también los propósitos formativos (más allá de los objetivos técnicos) que debe procurar lograr la asignatura.

${ }^{15}$ A manera de ejemplo, si bien todas las especialidades de la modalidad técnico-profesional consideran la enseñanza musical, existiendo también grandes diferencias con la modalidad humanísticocientífica (total horas HC: 240/4 años versus 120/4 años en todas las modalidades de enseñanza técnicoprofesional), la distribución de las horas en cada especialidad es diferente. En tal sentido, mientras que la cantidad de horas totales de educación musical para los cuatro años del plan general de la Enseñanza Comercial son 120, la cantidad de horas totales de educación musical para la Enseñanza Agrícola son 60, distribuidas en los mismos cuatro años. Al respecto, ver Decreto $N^{\circ} 11201,18 / 12 / 1967$. 


\section{Propósitos}

El currículo de 1965 posee un propósito definido para cada nivel y ciclo de enseñanza. Es así como en el primer ciclo el propósito es la "creación de oportunidades para el goce estético musical” del alumno. Este propósito se evidencia en una aproximación experiencial y lúdica a la música en la que el enfoque metodológico predominante es el de "descubrimiento" por parte del educando, y en el que debiera existir un predominio del "sentir" y el "hacer" musical por sobre una aproximación teórica, la que siempre debiera estar supeditada a la práctica musical. Por su parte, los ejes que conforman la propuesta son el canto individual y colectivo, el movimiento y la danza, la "audición controlada" y la creación y práctica de ejecución con instrumentos melódicos y de percusión.

El segundo ciclo tiene como propósito el "fortalecer la sensibilidad de los preadolescentes y adolescentes desarrollando su fantasía, imaginación creadora, apreciación estética, etc., impulsándolos a expresar su mundo interior y a la comprensión del trabajo y la recreación armónicamente combinados" 16 . El enfoque metodológico para este nivel distingue tres elementos, los cuales, ubicados en distintos niveles lógicos, dan cuenta de tres aproximaciones distintas y complementarias. Primero, una aproximación técnica y centrada en los contenidos, en la cual la canción constituye el núcleo del cual se desprenden los demás temas y contenidos. En segundo término, la organización de los contenidos musicales secuenciando el trabajo a partir del aprendizaje del ritmo, los aspectos melódicos y, finalmente, los aspectos armónicos en una aproximación teórica que destaca una creencia evolutiva del origen y desarrollo de la música en la civilización occidental, aplicada ahora a la enseñanza de la música. En tercer lugar, la mirada de la psicología educacional que organiza y orienta la aplicación del programa a partir del desarrollo de objetivos, conductas y contenidos. Finalmente, los ejes del currículo para este nivel son cuatro: canto, lectura entonada y escritura musical, audición de obras y estilos musicales además de creación musical.

La enseñanza media adapta los propósitos de acuerdo a la manera en que estructura la enseñanza en los dos subciclos. Es así como se identifica un propósito general, el cual, alineado con la mirada psicologista del currículo, busca en el plan general "contribuir al desarrollo integral de la personalidad del educando, por medio de la experiencia musical". Esto se reafirma con un mayor grado de especificidad en el plan diferenciado, en el que se busca "contribuir a la formación integral de la personalidad del educando a través de la experiencia consciente de la música”. Sin embargo, el plan diferenciado señala otros propósitos, entre los cuales se destaca el "perfeccionar al educando en el conocimiento de técnicas elementales y prácticas que le posibiliten una mejor comprensión de la música, al mismo tiempo que pueda desarrollar aptitudes y habilidades con miras a un futuro profesional o lo constituya en un auditor o consumidor de música, lo cual finalmente creará una mayor demanda musical"17. Esto apunta, además del desa-

${ }^{16}$ Programa de Educación Musical, segundo ciclo básico. Ministerio de Educación, 1968.

${ }^{17}$ Programa de Educación Musical de tercer año de enseñanza media, 1965, p. 48. 
rrollo de habilidades relativas a la práctica musical, a la formación de auditores informados, lo cual entrega una mirada amplia sobre la enseñanza de la música, que abarca tanto el ámbito de la producción (cantar, tocar instrumentos, componer) como también el de la reflexión y la apreciación estética de la música.

\section{ENSENANZA ESCOLAR DE LA MÚSICA EN LA REFORMA DE 1981}

\section{Antecedentes}

Los cambios políticos y sociales suscitados durante la década del 70 tienen particulares resonancias sobre el sistema escolar, el currículo, la formación docente y, en general, el campo educativo en su conjunto. Estas se traducen en un cambio abrupto de las reglas al interior del campo con la incorporación de principios económicos liberales como criterios de regulación al interior de las políticas, las instituciones y las demás agencias educativas en un marco de restricción de las libertades individuales y la disolución o suspensión de las agrupaciones sindicales y de profesionales ${ }^{18}$. Estos cambios pueden resumirse en tres grandes ámbitos: educación superior, currículum y gestión del sistema escolar.

En la educación superior, los principales cambios suceden a partir de la fragmentación de las dos universidades del Estado, la Universidad de Chile y la Universidad Técnica del Estado, ambas, hasta entonces, verdadera columna vertebral del sistema universitario nacional junto con la Universidad Católica. En segundo lugar, la reestructuración legal del sistema universitario en su conjunto, ahora compuesto de tres tipos de instituciones de formación superior, universidades, institutos profesionales y centros de formación técnica, ordenadas en un sistema estratificado y con diferentes características según el tipo de carreras que en ellos se imparten ${ }^{19}$. En lo que respecta a educación, son disueltos los institutos pedagógicos de Santiago y Valparaíso, los que se transforman en Academias Superiores de Ciencias Pedagógicas, al ser separados de la Universidad de Chile. Ellos pierden el estatus de institutos universitarios para pasar a formar parte de los institutos profesionales.

En el ámbito curricular se mantienen los lineamientos de 1965, aunque depurados de contenidos potencialmente conflictivos o politizados, orientándolos ahora hacia una visión tradicionalista de la enseñanza, inspirada en los principios humanista-cristianos presentes en la declaración de principios de la Junta Militar de 1974. En su matriz organizacional el currículo se reestructura a partir de principios neoliberales. Según Núñez, "los nuevos planes y programas se caracterizaron por preferir una indicación de objetivos o de conductas a lograr antes que una selección de contenidos, por dejar un amplio margen de opción metodológica a

\footnotetext{
${ }^{18} \mathrm{Al}$ respecto, ver Cox 1986.

${ }^{19}$ Esto redunda en una jerarquización explícita de las carreras asignadas a universidades, institutos profesionales y centros de formación técnica. Según Iván Núñez, "a las primeras, se reservaron doce carreras de alto prestigio y de mayor duración académica. Los institutos profesionales fueron definidos como entidades docentes que mantendrían carreras de cuatro o cinco años de duración, en tanto que los centros de formación técnica ofrecerían carreras técnicas cortas, de no más de dos años de duración" (Núñez 1997: 5).
} 
los profesores, y dentro de un marco determinado y un núcleo curricular mínimo, por permitir una importante flexibilidad a los establecimientos para estructurar el plan de estudios" (Núñez 1997: 6). Junto a esto se mantuvo la estructura de dos grandes niveles de ocho y cuatro años respectivamente, con cambios para la enseñanza media. Se establece un plan general común para la enseñanza media HC y la TP, con diversificación en $3^{\circ}$ y $4^{\circ}$ año medio. Otra innovación, también instaurada a partir de la Constitución de 1980, es la creación de instrumentos para la medición de los aprendizajes, lo cual da origen a la Prueba de Evaluación del Rendimiento (PER) entre 1982-1984, antecesora de las pruebas que componen el Sistema de Medición de la Calidad de la Educación (SIMCE), surgido en 1988.

Con respecto a la gestión del sistema educativo, tres grandes cambios se suscitan a partir de la segunda mitad de la década de 1970 con profundo impacto en el sistema educativo en su conjunto. Estos son el intervencionismo de la Junta Militar en el control y en la administración de la educación superior, la implementación de procesos de descentralización del Ministerio de Educación ${ }^{20}$ y la instauración de un nuevo sistema de financiamiento para la educación particular. Este último se inspiró en el concepto de subsidio educacional por alumno de Friedman ${ }^{21}$. No obstante, a diferencia del modelo original, fueron pagados mediante vouchers directamente a los sostenedores de los establecimientos, en base a la matrícula de las escuelas. Lo anterior redefinió las relaciones entre el Estado y la educación con importantes efectos en la equidad e igualdad de oportunidades de aprendizaje para los estudiantes del país y de condiciones laborales (contractuales y salariales) de los profesores y trabajadores del sistema escolar y educacional ${ }^{22}$. Los tres ámbitos generaron profundas consecuencias en el sistema escolar en su conjunto, y particularmente en la educación musical, en la cobertura y cantidad de horas, de sus fines y de sus propósitos, de sus contenidos y, finalmente, de su relación con la vida social del país.

\subsection{Currículum de educación musical 1981}

En el currículo de 1981 la educación musical mantiene su presencia durante el primer y segundo ciclo, asumiendo un carácter optativo en el plan general de la enseñanza media. Para el plan diferenciado de tercer y cuarto año medio la asig-

\footnotetext{
${ }^{20}$ Nos referimos a la creación de las secretarías regionales ministeriales, la entrega de la administración de gran parte de los establecimientos de formación técnico profesionales a corporaciones privadas; entrega de la administración de los establecimientos de enseñanza básica y media a las municipalidades.

${ }^{21}$ Cox 2003:25.

${ }^{22}$ Según Cox, el régimen militar redefine los límites institucionales externos del sistema educacional en una triple dirección, a partir de tres factores: municipalización (cambio en la relación del Estado con la administración educacional), privatización (apertura del sistema educativo a los principios del mercado) y traspaso de un sector de la enseñanza TP a las empresas (ligazón de la educación con la producción, especializando el currículum a partir de las necesidades de las empresas). Asimismo, redefine también los límites internos de la educación superior, estableciendo un sistema jerarquizado de tres niveles, con la fundación de dos modalidades de estudios postsecundario: institutos profesionales y CFT. Al respecto, ver Cox 1986.
} 
natura lectiva es sustituida por la realización de talleres en las áreas de folclore, instrumentos musicales, apreciación musical y coro. La estructura interna del currículo se sustenta sobre tres grandes elementos: los objetivos que persigue, los enfoques metodológicos y los contenidos asociados a cada objetivo. El currículo es claro en afirmar que más que proponer una serie de contenidos lo que busca es sentar una serie de metas a alcanzar al término del ciclo escolar. No obstante, tanto los objetivos que plantea como también las sugerencias metodológicas abordan explícitamente una serie de contenidos, los que mantienen en términos generales los lineamientos del currículum de 1965. Sin embargo, las diferencias se advierten al momento de buscar una estructura que, subyacente a los tres ciclos, los integre estableciendo progresión y continuidad en los aprendizajes. En este sentido, y a diferencia del currículo de 1965, el currículo de 1981 no posee ejes que ordenen los contenidos en una secuencia temporal de profundización de habilidades, o de amplitud en el tratamiento de contenidos.

Esto es visible al comparar, por ejemplo, los objetivos propuestos para el primer y segundo ciclo de enseñanza básica y para el plan general de enseñanza media. En los tres ciclos se aprecian contenidos relacionados entre sí, especialmente aquellos referidos a la enseñanza de la lectoescritura y los elementos básicos de la música. Sin embargo, no es posible encontrar alguna estructura que los ordene en una secuencia explícita entre los ciclos y niveles.

El musicólogo y académico Samuel Claro Valdés, entrevistado en el número 80 de la Revista de Educación, señala dos principales características de los programas de educación musical de 1980. Formalmente, se elimina el "burocratismo", representado a su juicio por las sugerencias metodológicas pormenorizadas. Conceptualmente, se parte de una proposición mínima, "pidiendo al profesor que los aplique con mayor profundidad en aquellas áreas que conoce mejor. Es decir, fija el límite inferior, pero no el superior" 23 . Junto con esto, señala que estos nuevos programas indican solo algunas pautas generales, lo cual los hace aparecer más reducidos. Sin embargo, en referencia a la aplicación de los programas, señala que "la correcta aplicación dependerá del profesor que los emplee. En este momento coexisten en educación general básica a lo menos profesores con dos tipos de formación" 24 , haciendo referencia a los profesores normalistas que siguen en el sistema y los profesores formados por las universidades desde la década del 60, estos últimos carentes a su juicio de una formación musical profunda. Señala a continuación: "por ello pienso que estos programas son bonitos, pero no tienen docentes preparados para llevarlos a cabo. En el futuro, el profesor básico debe contar con una formación musical más profunda, porque si no, ¿qué pasa? En una parte importante del país donde no haya docentes capacitados -y tal como el mismo programa lo indica- no se va a dar la clase de Música. Es un peligro que la ausencia de profesores idóneos se traduzca en la desaparición de la asignatura" 25 . Resulta interesante, por decir menos, la claridad de juicio del en-

\footnotetext{
${ }^{23}$ Revista de Educación, $\mathrm{N}^{\circ} 80$ (1980): 74-75.

${ }^{24} \mathrm{Ibid}$.

${ }^{25}$ Ibid: 75 .
} 
trevistado respecto a los riesgos que corría la enseñanza musical escolar en el período con la nueva estructura curricular y las características de formación del profesorado de la época.

\section{Propósitos}

Para el primer ciclo de enseñanza, los propósitos planteados apuntaban a "lograr un acercamiento a la música, preferentemente sensible, a través de la expresión vocal, instrumental, corporal, y por medio de la audición dirigida de un repertorio adecuado a su etapa de crecimiento y desarrollo y a sus intereses". A lo que agrego el "desarrollar una actitud sensible y crítica respecto a los estímulos provenientes del entorno, con el objeto de apreciar la belleza del marco cotidiano y evitar su progresiva contaminación sonora" ${ }^{26}$. Ambos propósitos buscan desarrollar experiencias formativas en los estudiantes más que una formación musical basada en contenidos.

Hilda Ruz, quien en 1981 estaba a cargo del área de Educación Musical del Centro de Perfeccionamiento, Experimentación e Investigaciones Pedagógicas (CPEIP), señala como el principal propósito de la educación musical en el primer ciclo de enseñanza básica el "producir momentos de felicidad en los niños: Hacer que los niños sean felices, participen con alegría, entusiasmo y satisfacción en las actividades propuestas para el logro de otras metas" 27 . Esto cambia durante el segundo ciclo, en el que los objetivos apuntan a "comprender y utilizar expresivamente los elementos básicos de la música y sus formas de organización (sonido, ruido, silencio, ritmo, melodía, armonía, forma y recursos expresivos), a través de la interpretación y aplicación integrada de ellos en canciones del repertorio docto, popular y folclórico, sensibilizarse en forma activa frente al fenómeno musical en sus estratos docto, popular y folclórico, como consecuencia de una apreciación analítica progresiva, y a incorporar los elementos ya conocidos en el estudio sistemático de las manifestaciones folclóricas nacionales y latinoamericanas, considerando los factores socioculturales que las condicionan" 28 . Con esto, se introducen directamente en el segundo ciclo básico la enseñanza de lenguaje musical, la práctica de la ejecución y la apreciación musical, y una relación más estrecha con el folclore nacional y latinoamericano, a partir de la integración de elementos socioculturales al análisis de los repertorios.

Los propósitos del plan general de enseñanza media se extienden hacia cuatro áreas, las que buscan "desarrollar capacidades para interpretar vocal y creativamente las canciones del repertorio coral escolar, expresando afinación melódica, justeza rítmica, fraseo musical, matices de dinámica y agógica, dicción e intencionalidad, contribuir al crecimiento espiritual del joven, desarrollando capacidades que le permitan enriquecer su sensibilidad artística, afectiva y social

\footnotetext{
${ }^{26}$ Programa de Educación Musical, Ministerio de Educación, en Revista de Educación, № 79 (mayo, 1980), pp. 102-106.

${ }^{27}$ Ruz 1981: 26-29.

${ }^{28}$ Programa de Educación Musical, Ministerio de Educación, en Revista de Educación, № 79 (mayo, 1980), pp. 102-106.
} 
a través de la expresión musical corporal, instrumental y mixta, desarrollar la capacidad audioperceptiva para apreciar, comprender, valorar y expresar con originalidad ideas, gustos, actitudes y juicios de valor respecto de la música y lograr mayor crecimiento de su sensibilidad artística, y estimular la creatividad en la expresión de sentimientos, ideas y estados anímicos aplicando los elementos que estructuran la música”, en un acercamiento más profundo a la expresión musical, al desarrollo de habilidades auditivas y al fomento de la creatividad musical.

Cabe hacer notar que en el análisis del programa de educación musical para los tres ciclos se advierte un marcado énfasis hacia los contenidos de folclore nacional y latinoamericano, tanto en lo referente a práctica de ejecución como de apreciación musical, así como notoria ausencia de referencias a otros repertorios específicos, consistente con la orientación nacionalista que inspira a esta reforma. Asimismo, llama la atención la importancia asignada a la enseñanza del Himno Nacional y otros de índole militar, los cuales, al igual que durante las primeras décadas de la República, hacen énfasis en consolidar una representación común de patria y de nación en el estudiantado.

\section{ENSEÑANZA ESCOLAR DE LA MÚSICA EN LA REFORMA DE 1996-1998}

\section{Antecedentes}

En marzo de 1990, un día antes de finalizar el gobierno militar, es aprobada la Ley Orgánica Constitucional de Enseñanza (LOCE), contemplada desde la reforma de 1981, y formulada y aprobada en las postrimerías del régimen militar. Dicho marco legal modifica en forma sustantiva la estructura curricular y el rol de las instituciones del sistema escolar nacional, incluyendo el Ministerio de Educación. En efecto, la aprobación de la LOCE implicó un cambio profundo para la arquitectura del sistema educacional en su conjunto. Define un nuevo "orden institucional”, con la creación del Consejo Superior de Educación (CSE), organismo compuesto por autoridades provenientes de diversas instituciones del país -incluyendo las Fuerzas Armadas y la Corte Suprema- que tiene como principal fin el resguardo de la acción del Ministerio de Educación, buscando mantener bajo control el accionar del Estado en materia de educación ${ }^{29}$.

Dentro de las atribuciones de la LOCE estuvo la creación de un nuevo currículum, basado en un Marco Curricular ${ }^{30}$ que estableció objetivos fundamentales y contenidos mínimos obligatorios, además de planes y programas de estudio para cada una de las áreas que componen el currículo, lo cual es realizado a partir de los proyectos MECE básica (1996) y MECE media (1998). Las características de la nueva arquitectura curricular, similar a otras desarrolladas por países de avanzada en materia de educación, tales como Australia y el Reino Unido, entre otros, permite el desarrollo de un sistema curricular mixto. En él, el Estado establece, a partir de un principio de equidad, una selección de competencias, habilidades, destrezas y conocimientos que deban ser desarrollados en todos los establecimien-

\footnotetext{
${ }^{29} \mathrm{Al}$ respecto, ver Gysling 2003: 213-252, Cox 2003a.

${ }^{30}$ Decreto Supremo No 220, 1998, Santiago: Ministerio de Educación.
} 
tos escolares del país. A la vez reconoce la posibilidad de que los establecimientos desarrollen sus propios planes y programas de enseñanza, los que, cubriendo las definiciones de Objetivos Fundamentales y Contenidos Mínimos Obligatorios, les permita implementar propuestas a partir de sus propias y particulares orientaciones formativas.

En cuanto a sus rasgos fundamentales el nuevo currículum define cuatro dimensiones de cambio: "i) las relaciones de control del mismo; ii) las características de su arquitectura mayor o estructura (qué secuencia de años, organizada cómo; qué distinciones dentro de tal secuencia); iii) su organización en espacios curriculares determinados dentro de tal estructura (¿hay nuevas asignaturas, o redefinición de relaciones entre las mismas?); iv) cambios de orientación y contenidos dentro de tales espacios curriculares, áreas, o asignaturas" ${ }^{31}$.

En términos culturales, tres principios orientan la construcción del nuevo currículo: “a) la necesidad de actualizar la experiencia curricular en relación con los cambios epocales que está experimentando la sociedad; b) la necesidad de mejorar la calidad de una experiencia educacional no solo inequitativa, sino que empobrecida en sus expectativas para el conjunto de la matrícula escolar; c) la necesidad de 'modernizar' la base valórica de la sociedad, que viene saliendo de 17 años de gran repliegue cultural, y reinstalar en la educación los valores democráticos" ${ }^{32}$.

Respecto a su estructura interna, el Marco Curricular se conforma a partir de Objetivos Fundamentales Verticales, relacionados con los saberes disciplinarios, y de Objetivos Fundamentales Transversales, los que a su vez tienen conexión con saberes de orden valórico y ético principalmente vinculados con la formación moral y ciudadana de los estudiantes. El Marco Curricular define además los Contenidos Mínimos Obligatorios, es decir, un piso de conocimientos que deben ser abordados en todos los establecimientos escolares del país, para cada uno de los niveles o cursos. Dentro de los cambios que se suscitaron en el contexto histórico de creación del nuevo currículum, está la promulgación del Estatuto Docente (el que permitió una relación más cercana con el Colegio de Profesores, de importancia capital para la implementación de las nuevas políticas relacionadas con la implementación de la Reforma), la creación de nuevos instrumentos (Marco para la Buena Enseñanza, entre otros) y la posterior implementación de la Evaluación Nacional Docente.

\subsection{Currículum de Artes Musicales 1996-1998}

Uno de los cambios propuestos por la Reforma afecta a la denominación de la asignatura, ahora denominada Artes Musicales. Este cambio se enlaza con el cambio de arquitectura curricular, en la cual ya no existen asignaturas, sino que se reconocen áreas del conocimiento denominadas "sectores de aprendizaje". En este ámbito, la asignatura forma parte del sector de Educación Artística (obligato-

\footnotetext{
${ }^{31}$ Cox 2003b:3.

${ }^{32}$ Gysling 2003: 218.
} 
rio para la Formación General), compuesto por Artes Musicales, Artes Visuales y nominalmente por Artes Escénicas ${ }^{33}$. Otro de los cambios corresponde a la organización de las horas al interior del currículum de enseñanza básica y media, en la cual incide directamente la actual categoría de "subsector" que posee. El currículo de 1996-1998 define un número de horas destinado a la Educación Artística, el cual varía de acuerdo al ciclo y nivel en que se encuentre. El siguiente cuadro presenta el plan de estudios para el sector de Educación Artística durante los 12 años de escolaridad básica y media.

PLAN DE ESTUDIOS, SECTOR DE EDUCACIÓN ARTÍSTICA

\begin{tabular}{|c|c|c|c|}
\hline Niveles & \multicolumn{2}{|c|}{ Horas } & Áreas de cobertura \\
\hline $\begin{array}{c}\mathrm{NB} 1 \\
\left(1^{\circ} \text { y } 2^{\circ} \text { básico }\right)\end{array}$ & \multicolumn{2}{|c|}{3} & $\begin{array}{l}\text { Artes Musicales, Artes Visuales, Artes } \\
\text { Escénicas. }\end{array}$ \\
\hline $\begin{array}{c}\mathrm{NB} 2 \\
\left(3^{\circ} \text { y } 4^{\circ} \text { básico }\right)\end{array}$ & \multicolumn{2}{|c|}{4} & Artes Musicales, Artes Visuales. \\
\hline $\begin{array}{c}\text { NB3 } \\
\left(5^{\circ} \text { básico }\right)\end{array}$ & \multicolumn{2}{|c|}{3} & Artes Musicales, Artes Visuales. \\
\hline $\begin{array}{c}\mathrm{NB} 4 \\
\left(6^{\circ} \text { básico }\right)\end{array}$ & \multicolumn{2}{|c|}{3} & Artes Musicales, Artes Visuales. \\
\hline \multirow{2}{*}{$\begin{array}{c}\text { NB5 } \\
\left(7^{\circ} \text { básico }\right)\end{array}$} & \multirow{2}{*}{4} & 2 & Artes Musicales. \\
\hline & & 2 & Artes Musicales. \\
\hline \multirow{2}{*}{$\begin{array}{c}\text { NB6 } \\
\left(8^{\circ} \text { básico }\right)\end{array}$} & \multirow{2}{*}{4} & 2 & Artes Visuales. \\
\hline & & 2 & Artes Visuales. \\
\hline NM1 & \multicolumn{2}{|c|}{2} & Artes Musicales o Artes Visuales. \\
\hline NM2 & \multicolumn{2}{|c|}{2} & Artes Musicales o Artes Visuales. \\
\hline NM3 & \multicolumn{2}{|c|}{2} & Artes Musicales o Artes Visuales. \\
\hline NM4 & \multicolumn{2}{|c|}{2} & Artes Musicales o Artes Visuales. \\
\hline
\end{tabular}

Se observa presencia obligatoria del sector de Educación Artística a lo largo de todo el currículo, pero, bajo un principio de optatividad, el que es también común a la mayoría de los niveles. Este punto, así como los aspectos relativos a la clasificación interna de los conocimientos del sector de Educación Artística, serán abordados más adelante.

\footnotetext{
${ }^{33}$ Decimos nominalmente porque, a pesar de formar parte de las definiciones establecidas en el Marco Curricular, no existen planes ni programas que definan aprendizajes para esta área.
} 
La estructura interna del currículum de Artes Musicales mantiene una estructura general que es común para la Educación Artística, la cual se configura a partir de tres ejes básicos: producción, apreciación y reflexión. El primero corresponde a la puesta en acto de la expresión artística, interpretación y creación en el caso de la música. El segundo corresponde al desarrollo de capacidades de percepción y apreciación estética. Por su parte, el tercero apunta al desarrollo de capacidades de reflexión crítica a partir del análisis de obras, productos o fenómenos artísticos. En la enseñanza media, se especifica además para la música la existencia de dos dimensiones complementarias: la música como disciplina artística y la música como cultura. A través de estas dimensiones se busca integrar la visión de la música en tanto práctica artística con la dimensión social de la música, inserta en la sociedad y la cultura. Asimismo, son indicados tres ámbitos específicos de la práctica musical, los que organizan la experiencia a partir de la interpretación, la creación y la apreciación musical.

\section{Propósitos}

Los propósitos que busca alcanzar el currículo de educación artística en el primer ciclo básico son el "desarrollar en el estudiante la capacidad de expresión artística e iniciar la percepción estética del entorno y la apreciación de obras de arte"34, introduciendo al alumno en la experiencia artística a partir del desarrollo de la expresión y la apreciación estética. Para el segundo ciclo de Educación Artística, se busca "desarrollar en el alumno la capacidad de expresión y apreciación artística en relación con diferentes temáticas y lenguajes del Arte, así como la comprensión, en un nivel básico, de acontecimientos de la historia del arte"35. Continúa la experiencia iniciada en el primer ciclo expandiéndose hacia nuevas temáticas y lenguajes junto con relacionar en un nivel primario la música con sus características socioculturales, en una preparación para abordar de una forma más comprensiva el desarrollo de la apreciación estética y la reflexión.

Los dos últimos años del segundo ciclo básico coinciden con los dos primeros años del subsector de Artes Musicales. Este cambio se percibe no solo en relación a la nomenclatura, sino también en la orientación que comienza a tomar el subsector, desmarcándose de la matriz común de Artes, para desarrollar habilidades y unidades de conocimientos propios al conocimiento musical. Los propósitos para Artes Musicales en NB5 y NB6 son el "expresarse por medio de la voz y los instrumentos en torno a diversos repertorios”. A esto se agrega el desarrollo de habilidades de discriminación auditiva, sumado al conocimiento de variados repertorios, estilos y manifestaciones musicales, en sintonía con los dos grandes propósitos planteados para la educación básica.

Por otra parte, los propósitos de la educación media, organizados en cuatro ejes temáticos (percepción, lenguaje musical, apreciación-reflexión, diseño de proyectos), buscan desarrollar, respectivamente, aprendizajes en el ámbito de la

\footnotetext{
34 Objetivos Fundamentales y Contenidos Mínimos Obligatorios de la Educación Básica, p. 155.

${ }^{35}$ Ibid., p. 155-156.
} 
percepción sonora y la audición musical. Consignan, además, el desarrollo de conocimientos de lenguaje musical (tanto en interpretación como en composición, principalmente desde el análisis de los recursos de expresión y construcción musical), el goce estético mediante la práctica de ejecución y creación, la indagación en las relaciones entre música, cultura y sociedad (considerando las relaciones con las músicas tradicionales, la historia y el desarrollo de los estilos, la industria, entre otros), junto al diseño y ejecución de proyectos que integren diversos conocimientos (estéticos, tecnológicos, organizacionales), con el fin de difundirlos hacia la comunidad inmediata.

Dentro de los énfasis que el currículo de 1996-1998 realiza, destaca esta nueva relación entre música y cultura, visible en la amplitud con que aborda los repertorios, como también en las diversas categorías en que los organiza, v. gr. estilos, géneros, períodos de la historia musical, distribución geográfica. Asimismo, el abordaje de temáticas que desarrollan esta relación entre música y cultura aparece desde el segundo ciclo básico, primero con la organización de las unidades a partir de la realidad local del alumno hasta cubrir las prácticas musicales en contextos de amplitud creciente, comunidad inmediata, región, Chile y Latinoamérica, resto del mundo.

\section{CONCLUSIONES}

En el intento de llegar a una mirada sintética a partir de la comparación entre los currícula, hemos privilegiado el análisis en dos ámbitos, de los cuales emergen dos grandes categorías: características de la clasificación de la asignatura de enseñanza musical en cada currícula y caracterización de los contenidos de cada uno. El primer ámbito toma como punto de partida el concepto de clasificación desarrollado por el sociólogo británico Basil Bernstein (1974), para describir las características principales de cada currículo a partir de la naturaleza de la diferenciación entre los contenidos. Señala Bernstein que la "clasificación, se refiere al grado de mantenimiento de los límites entre contenidos”, es decir, donde la diferenciación no es parte de los propios contenidos, sino que se observa a partir de los límites existentes entre dichos contenidos. Según el autor, es posible distinguir dos tipos de clasificación: clasificación fuerte, en la que "los contenidos están claramente aislados los unos de los otros por fuertes límites”, y clasificación débil, cuando existe un aislamiento reducido entre los contenidos, pues las fronteras entre éstos son débiles o están borradas ${ }^{36}$.

El segundo ámbito dice relación con los contenidos propiamente tales, los cuales, al ser mirados en perspectiva, evocan las concepciones sobre música y enseñanza musical que cada currículo estableció como base en su selección de conocimientos. De esta forma, los énfasis y enfoques que desarrolla cada currículo en términos de saber disciplinario constituyen elementos que reflejan conceptualizaciones, creencias y valoraciones acerca de la música en nuestra sociedad.

\footnotetext{
${ }^{36}$ Bernstein 1974 [1985]: 37.
} 
En términos clasificatorios, el currículo de 1965 posee límites visibles entre la Educación Musical y las Artes Visuales, con contenidos claramente definidos y correspondientes a la disciplina junto a una clara ordenación de los saberes que contiene, fruto de los ejes que lo estructuran, lo cual representa una clasificación fuerte. En la misma categoría, el currículo de 1981 plantea un discurso amplio, en el que se distinguen algunos ámbitos de saber disciplinar, distintos de las otras artes, pero en un contexto de clasificación débil. La estructura curricular carece de ejes que sustenten la progresión y la secuenciación del aprendizaje. Además, no se explicitan los contenidos para los objetivos planteados, los que pueden ser cambiados, sustituidos e incluso omitidos en la práctica, merced a los principios de regulación establecidos en el propio currículo. Para la misma categoría, el currículo de 1996-1998 se comporta en forma diferente de acuerdo al ciclo, primer y segundo ciclo de enseñanza básica, ciclo de enseñanza media, y en la relación entre sector y subsector. Para el primer ciclo básico el currículo posee una clasificación débil, con límites difusos entre los objetivos comunes para las dos áreas y contenidos que no logran diferenciarse totalmente entre unidades y niveles al interior del ciclo, el que tiene una marcada impronta de las Artes Visuales tanto en el diseño de los aprendizajes como en los fines explicitados para todo el ciclo. En el segundo ciclo básico los límites se hacen más fuertes, coexistiendo aun principios comunes pero con una mayor definición de los límites entre contenidos. Sólo en la segunda mitad del ciclo básico, NB5 y NB6, equivalentes al $7^{\circ}$ y $8^{\circ}$ año básico, así como para todo el ciclo de enseñanza media, se observa una clasificación fuerte, con límites marcados y diferenciados entre las Artes Musicales y las otras artes, con objetivos y contenidos formulados a partir de criterios explícitos de secuencia y progresión.

En términos de contenidos, el currículo de 1965 define unos ejes o dimensiones que estructuran la experiencia musical de los alumnos. De esta forma, el currículo comenzaba por presentar dos ejes -amplios y principalmente enfocados al desarrollo de la experiencia musical- los cuales se desarrollan a través de los diferentes ciclos, dentro de relaciones de secuencia. La amplitud de los contenidos abordados a lo largo de todo el currículo se refleja en la inclusión de repertorios de diversas procedencias, docto, folclórico y popular, además de diversos estilos y épocas. Se observa además que tal amplitud se restringe en su base, pues ésta asume una matriz de pensamiento ligada a la música tradicional europea que establece el canon que regula las relaciones con los demás repertorios. Esto se reafirma con el énfasis que el currículo de 1965 otorga al aprendizaje del lenguaje musical formal por medio de la práctica musical, la lectoescritura, la audición dirigida y la creación.

Por su parte, el currículo de 1981 mantiene tres ámbitos de repertorio pertenecientes al currículo anterior, docto, folclórico y popular, en el que han sido eliminados los contenidos que dan cuenta de relaciones culturales entre el lenguaje y la práctica musical. De esta forma, el currículo de 1981 prescribe unos contenidos para ser implementados en situación de contingencia, en la que no es posible establecer una secuencia con otros aprendizajes a partir de lo prescrito, sino en función de conectar exclusivamente aspectos de lenguaje, principalmen- 
te notacionales y de forma, con la práctica musical directa, sin establecer nexos con los principios de generación y transmisión social que cada repertorio posee. Asimismo, los contenidos relativos al repertorio son categorizados e implícitamente jerarquizados en la propuesta curricular en función de criterios extramusicales. De este modo, el repertorio folclórico se asocia con principios de patria y nación, mientras que los repertorios de música docta, nombrados en el currículo de 1981 como "de Arte Superior", y la música popular se asocian a principios de alta y baja cultura, respectivamente. Todo lo anterior derivó en una propuesta curricular que mantiene el énfasis por la enseñanza del lenguaje musical, ahora entendido como una práctica estrictamente notacional, sin producción de crítica ni discursos, y sujeto a un marco estructural difuso en términos de relaciones entre de contenidos y aprendizajes.

En términos de contenidos, el currículo de música de 1996-1998 se caracteriza por abordar dos ámbitos del conocimiento musical. Por una parte, los saberes relativos a la música como disciplina artística, esto es, contenidos que se relacionan con las prácticas musicales de audición, interpretación y creación, los cuales consideran aspectos relativos al lenguaje musical -lectoescritura, conceptos musicales, taxonomías, etc.- y a la ejecución instrumental y vocal, principalmente de tipo procedimental. Por otra parte, figuran los conocimientos relativos a la música como cultura que considera principalmente el establecimiento de relaciones discursivas y procedimentales entre la producción de música, la cultura y la sociedad, vinculadas principalmente con saberes relativos al medio ambiente sonoro, la apreciación estética, el desarrollo tecnológico, los repertorios de diversas proveniencias, la identidad musical y el patrimonio, las relaciones entre música e industria y la reflexión acerca de los usos y funciones de la música en la sociedad.

Lo anterior descansa sobre una estructura de tres ejes, que categoriza los conocimientos en tanto producción, apreciación y reflexión, estructura que articula todo el currículo de Artes, considerando las Artes Visuales.

Cabe mencionar que el currículo de música de 1996-1998 se enfoca fuertemente en el desarrollo del lenguaje musical. No obstante, a diferencia de los dos anteriores, establece su foco no en la adquisición de una mirada particular basada en la música de tradición europea, ni en la práctica de unas destrezas de lectoescritura. Se pretende más bien que el lenguaje musical desarrolle competencias que permitan, a partir de la práctica musical -de percepción, apreciación, interpretación y creación- generar un pensamiento reflexivo que apunte a la producción de un discurso musical informado y crítico.

El cuadro de la página siguiente resume las características de cada currícula, en las tres categorías analizadas.

Con respecto a los repertorios abordados, es posible realizar observaciones sobre criterios de secuencia entre ellos. En tal sentido, se observa que sólo el currículo de 1965 establece una ordenación sobre la práctica -de audición y de interpretación- de los repertorios. La prescripción, durante la enseñanza media, de ordenar los repertorios en función del interés de los alumnos y no solo a partir de un criterio histórico, establece una diferencia con los demás currícula en los cuales no se prescribe el orden en que deben ser abordados. Se observa, además, 


\begin{tabular}{|c|l|l|l|}
\hline Categorías & \multicolumn{1}{|c|}{1965} & \multicolumn{1}{c|}{1981} & \multicolumn{1}{c|}{$1996-1998$} \\
\hline Clasificación & $\begin{array}{l}\text { Clasificación fuerte, } \\
\text { límites fuertes. }\end{array}$ & $\begin{array}{l}\text { Clasificación débil, } \\
\text { límites difusos. }\end{array}$ & $\begin{array}{l}\text { Clasificación débil, con } \\
\text { límites difusos en } \\
\text { básica; clasificación } \\
\text { fuerte, con límites } \\
\text { definidos en ed. media. }\end{array}$ \\
\hline \multirow{6}{*}{ Contenidos } & $\begin{array}{l}\text { Definidos por ejes, con } \\
\text { amplitud de reperto- } \\
\text { rios, construidos sobre } \\
\text { una matriz que } \\
\text { enfatiza el lenguaje de } \\
\text { la tradición docta } \\
\text { europea. }\end{array}$ & $\begin{array}{l}\text { Ausencia de ejes, } \\
\text { fuerte presencia de } \\
\text { repertorio tradicional } \\
\text { folclórico; fuerte } \\
\text { énfasis en lo nacional, } \\
\text { y en la enseñanza de } \\
\text { elementos de } \\
\text { lectoescritura musical. }\end{array}$ & $\begin{array}{l}\text { Repertorio amplio, } \\
\text { con énfasis en los } \\
\text { repertorios tradiciona- } \\
\text { les y populares, } \\
\text { además de una fuerte } \\
\text { reflexión sobre las } \\
\text { relaciones entre } \\
\text { música y sociedad; } \\
\text { organizados en dos } \\
\text { ámbitos, sobre 3 ejes. }\end{array}$ \\
\hline
\end{tabular}

que las causas que explican esta constatación son muy diferentes. En el currículum de 1981 no se especifican los contenidos -en este caso, repertorios- por obedecer a una lógica liberal en la gestión e implementación del currículo. Por otra parte, en la reforma de 1996-1998 esto podría explicarse a partir de una relación de horizontalidad existente entre los repertorios, ligada profundamente con el concepto de música como cultura subyacente a todo el currículo. Este concepto implica un cuestionamiento profundo a la jerarquización de los repertorios y a una posible secuenciación de ellos en términos de mayor/menor relevancia, mayor/menor complejidad, etc.

La profundidad con que se abordan los repertorios guarda relación indirecta con el currículo, obedeciendo más bien a la complejidad de los contenidos asociados a cada repertorio, planteados en las unidades proyectadas para cada ciclo y año. De esta forma, la profundidad con que se deben abordar los repertorios descansa, por un lado, en el o los criterios que aplica el profesor en la selección e implementación de repertorios para trabajar los contenidos. Por otro lado, guarda relación con los materiales en que se apoya el docente que forman parte del contexto social de cada currículo -textos de educación musical, métodos, colecciones de repertorio, publicaciones, registros de música, etc.- y que también se encuentran ligados a los criterios de selección empleados por el docente. Esto es común para los tres currícula.

La diferenciación en la clasificación constituye un punto crítico del currículo de 1996-1998 que se evidencia en dos grandes aspectos. Primero, en un mayor peso de las Artes Visuales en la generación de los Objetivos Fundamentales Verticales (OFV) y los Contenidos Mínimos Obligatorios (CMO) de enseñanza básica, ante los cuales los OFV y CMO de música, además de encontrarse en distinto nivel lógico, son adaptados de manera forzada. Segundo, y a consecuencia del peso 
que tienen las Artes Visuales en la arquitectura curricular de enseñanza básica, se observa una superposición de estructuras, en la que oficialmente se organiza la duración de la escolaridad en ocho y cuatro años respectivamente, mientras que en la definición curricular opera como dos niveles de seis años cada uno.

La introducción de las relaciones entre música y cultura establece una orientación temática distinta al interior del currículo -ligada a la musicología de enfoque antropológico, la que es concordante además con los principios culturales que orientan el currículo general. Esto plantea poderosos desafíos al momento de querer visibilizar la naturaleza de estas relaciones, no solamente al nivel de temáticas sino al de estructuras de relaciones, capaces de abrir a la discusión nuevos ámbitos de relación con la música y de explicar la forma en que se interrelacionan. Esto último no depende tanto de la capacidad discursiva con que se argumente cada nueva relación, sino que con la necesidad de una matriz teórica definida y explícita, que vaya más allá de unas orientaciones metodológicas y que otorgue una cualidad epistémica visible y explícita acerca de la orientación que desarrolla nuestro currículo.

\section{ACERCA DE LOS PROPÓSITOS Y EL SENTIDO DE LA ENSEÑANZA MUSICAL EN LA SOCIEDAD}

El análisis de los propósitos para los tres períodos revisados permite observar tres miradas, las que dan cuenta de tres sentidos distintos sobre la necesidad y finalidad de la enseñanza musical escolar en nuestra sociedad. Para el primer período (reforma de 1965), se plantea una educación musical capaz de contribuir al desarrollo completo de la persona en una dimensión en que las demás disciplinas no alcanzan a abordarlo: la dimensión expresiva propia del arte musical. Esta es sostenida por una amplia selección de conocimientos, proveniente de diversos ámbitos de la cultura, en la que predomina, en desmedro de otras, solo una matriz de pensamiento -el lenguaje musical del repertorio tradicional europeo, o docto. Su finalidad es la de formar personas que, habiendo vivenciado la experiencia musical y accedido a conocimientos específicos y particulares de la disciplina musical, puedan desarrollar una carrera musical con posterioridad a la formación escolar, o bien constituirse en auditores informados y capaces de apreciar la música sobre una base mínima de conocimiento musical.

En el segundo período, reforma de 1981, se observa una mirada que excluye la dimensión social de la música. No obstante, se releva desde el discurso la importancia que esta dimensión tiene en términos de formación integral para los alumnos, pero sin que la prescripción cuente con un corpus de conocimientos que sostenga esta mirada, ya sea por ausencia de importantes dimensiones relativas al saber musical, ya sea por la falta de una estructura que evidencie el desarrollo de ese aspecto formativo a lo largo del currículo. Éste, por otro lado, pareciera enfocarse en el desarrollo de un goce estético superficial, en el que propósitos enunciados se diluyen frente a las condiciones restrictivas impuestas por el contexto.

En el tercer período (reforma de 1996-1998) se constata una formación musical centrada en la persona, con un fuerte énfasis en la dimensión social de la 
música de la que se releva su presencia a nivel cultural. Se observa, además, la importancia que tiene tal dimensión en la comprensión de la nueva realidad postglobalización y en la adquisición de competencias que, formando parte de la arquitectura curricular mayor, buscan preparar a los estudiantes para los desafíos que imponen los nuevos tiempos. En el intento de una visión sintética -asumiendo el riesgo de ser además estrecha- observamos que esta propuesta se plantea como formadora de personas con criterio reflexivo, capacidades de apreciación y goce estético junto a una potencial capacidad de elaboración de discursos sobre la práctica musical, los usos y funciones de la música y las relaciones que ésta sostiene con la sociedad en su conjunto. Sin embargo, también es posible advertir que las bases teóricas en que se plantean estas nuevas relaciones entre música, cultura y sociedad no son explicitadas en la propuesta, tendiendo a desdibujar las orientaciones que, en el fondo, sustentan esta nueva visión del currículo. A esto se le suma una estructura curricular que, en términos de obligatoriedad, tampoco asegura la cobertura de esta mirada sociocultural de la música, ni su desarrollo en todos los niveles en que se imparte el subsector de Artes Musicales ${ }^{37}$.

Fuera del análisis quedan algunos elementos ajenos al objeto, que podrían complementar la visión expuesta en este texto y que enunciamos a manera de epílogo. Las relaciones entre currículo escolar con la formación de profesores, tanto en una mirada diacrónica como sincrónica, constituyen un elemento crucial para el análisis y la comprensión de la génesis y evolución de la enseñanza musical escolar en cada período. Asimismo, la profundización en el conocimiento de las relaciones entre la enseñanza musical escolar con el contexto sociopolítico mayor permite afinar la mirada sobre dicha génesis y evolución, estableciendo una conexión entre lo micro y lo macro a nivel de políticas educacionales, discursos y acción. Sumado a esto, la comprensión de aspectos relativos a la implementación curricular y las ideas de base del profesorado, en tanto concepciones, creencias y actitudes hacia el currículo y hacia la enseñanza musical, permitirían avanzar en una mirada profunda sobre la enseñanza musical escolar desarrollada en Chile durante los últimos 40 años.

Por otra parte, las relaciones entre música y sociedad pueden ser aún profundizadas, estableciendo relaciones entre los contextos en que fue planteada cada una de las reformas -incluyendo sus condiciones generativas y las características de los agentes y agencias que componen cada contexto- y los contenidos de música seleccionados para cada currículum. Consideramos que, por medio de un adecuado contraste entre el nivel macro y el nivel micro, es posible acercarse a una configuración sobre la función social que cumple la escuela y específicamente la clase de música en cada contexto histórico. Esta función es capaz de revelar los rasgos culturales de un tipo de persona que la sociedad desea formar, y que la enseñanza musical escolar debe ser capaz de reflejar al interior de cada currículo, en cada período histórico.

\footnotetext{
${ }^{37}$ Acerca de la obligatoriedad del subsector de Artes Musicales, se sugiere revisar las horas prescritas para el subsector en el cuadro Plan de Estudio, Sector de Educación Artística, presentado anteriormente.
} 


\section{BIBLIOGRAFÍA}

AlveAr, José

1996 Análisis y proyecciones del Proyecto MECE. Tesis para optar al título de Profesor de Educación Musical. Santiago: Universidad Metropolitana de Ciencias de la Educación (UMCE).

Aravena, Julio

1996 Análisis de las cohortes 1981-1985 de alumnos egresados de la carrera de educación musical de la Universidad Metropolitana de Ciencias de la Educación. Tesis para optar al título de Profesor de Educación Musical. Santiago: UMCE.

Bernstein, BASIL

1974 [1985] "On the Classification and Framing of the Educational Knowledge", Class, Codes and control. Volumen 3. Towards a Theory of Educational Transmissions. Londres: Routledge and Kegan Paul, 1974. Traducción de Mario Díaz, en Revista Colombiana de Educación, $\mathrm{n}^{\circ}$ 15, 1985, pp. 35-36.

1988 Clases, códigos y control, Vol. 2. Madrid: Akal.

Bourdieu, Pierre

1991 La distinción: criterios y bases sociales del gusto. Madrid: Taurus.

BRUNNER, José JOAQUín

1980 Sociología de los principios educativos: un análisis de dos reformas de los planes y programas de la enseñanza básica chilena: 1965 y 1980. Programa FLACSO (Facultad Latinoamericana de Ciencias Sociales)-Santiago de Chile, ${ }^{\circ}$ 95, (agosto). Santiago: FLACSO.

Brunner, José Joaquín, Hernán Courard, Cristián Cox

1992 "La educación superior en Chile, 1960-1990: evolución y políticas", Estado, mercado y conocimiento: politicas y resultados en la educación superior chilena, 1960-1990. Santiago: FLACSO, pp. 3-123.

Cox, CRISTIÁN

1986 Políticas educacionales y principios culturales, Chile 1965-1985. Santiago: Centro de Investigación y Desarrollo de la Educación (CIDE).

2001 "El currículum escolar del futuro", Revista Perspectivas, IV/2, publicación trienal del Departamento de Ingeniería Industrial, Universidad de Chile, pp. 213-232

2003a "Las políticas educacionales de Chile en las dos últimas décadas del siglo XX", Políticas educacionales en el cambio de siglo: la reforma del sistema escolar de Chile. Santiago: Editorial Universitaria, pp. 19-133.

2003b "El nuevo currículum del sistema escolar". Tomado de internet el 10 de enero de 2010, desde el sitio http://mt.educarchile.cl/mt/jjbrunner/archives/2008/01/ cristian_cox_ed.html.

Cox, Cristián y JacQueline Gysling

1987 La formación del profesorado en Chile. Santiago: CIDE.

ERRÁZuRIZ, LUIS HERNÁN

1994 Historia de un área marginal. Historia de la enseñanza artística en Chile 1797-1993. Santiago: Ediciones de la Pontificia Universidad Católica de Chile.

2004 "La educación artística en el sistema educacional chileno". Tomado de internet en agosto de 2009 desde http://portal.unesco.org/culture/en/ev.phpURL_ID=19709\&URL_DO=DO_PRINTPAGE\&URL_SECTION=201.html, 
FLICK, UwE

2004 Introducción a la metodología cualitativa. Madrid: Morata.

Grebe, María Ester

1975 "Preferencias musicales de la juventud escolar de Santiago" (primera parte), Revista de Educación, $\mathrm{N}^{\circ} 52, \mathrm{~N}^{\circ} 53, \mathrm{~N}^{\circ} 54$ (junio), pp. 73-79.

GySLING, JACQueLine

2003 "Reforma curricular: itinerario de una transformación cultural", Políticas educacionales en el cambio de siglo. Santiago: Editorial Universitaria, pp. 213-252.

Leyton, Mario

1968 "La reforma de la enseñanza media y sus proyecciones", Revista de Educación (nueva época), № 4 (marzo), Santiago: Ministerio de Educación Pública, pp. 6-17.

NÚÑEZ, IvÁN

1997 "Historia reciente de la educación chilena". Tomado de Internet el 16 de abril de 2010, desde el sitio http://historiaeducacion.tripod.com/sitebuildercontent/ sitebuilderfiles/historia_reciente.doc

NúÑEZ, JosÉ ABELARdo

1883 Organización de las Escuelas Normales. Santiago: Imprenta de las Librerías Americanas.

OTTENBERGER, ANNA

1983 Concepciones y vacios de la enseñanza de la música en la escuela primaria fiscal, . Chile: 1900-1950. Tesis para optar al grado de Licenciado en Ciencias de la Educación. Santiago: Pontificia Universidad Católica de Chile.

Pardo, María Teresa y Silvia Peña Hen

1960 Panorama de la educación musical en Chile y aporte de los compositores nacionales. Memoria para optar al título de Profesor de Estado en Música. Santiago: Universidad de Chile.

Ruz, HILDA

1980 "La música en el mundo del niño", Revista de Educación, № 77 ( febrero-marzo) (segunda edición), pp. 24-27.

1981 “Educación Musical”, Revista de Educación, Nº 87 (junio), pp. 26-29.

Santa Cruz, Domingo

1932 "El más grave problema de la música en los colegios". Aulos, año I/2 (noviembre), pp. 1-3.

1960 "La enseñanza de la música en Chile”, El estado actual de la educación musical en el mundo. Editado por Egon Kraus. Buenos Aires: EUDEBA, pp. 46-69.

Sepúlveda, Ana Teresa

1996 Presencia de la música en la enseñanza secundaria chilena: una visión histórica a través de 5 reformas educativas 1893, 1935, 1955, 1965, 1981. Tesis para optar al grado de Doctor en Educación. Salamanca: Universidad Pontificia de Salamanca.

Vicuña, Magdalena

1980 "La educación musical del niño", Revista de Educación, № 80 (junio-julio), pp. 69-70. 


\section{DOCUMENTOS}

ChILE

Planes y PROGRAMASS

1965

Programas de educación musical, Revista de Educación $\mathrm{N}^{\circ} 12$ (segunda edición), número especial con los programas de educación básica (agosto, 1969), pp. 247-259.

Programa de educación musical, primero año medio, Revista de Educación $\mathrm{N}^{\circ} 5$ (nueva época) número extraordinario con los programas de estudio del primer año de enseñanza media (abril, 1968), pp. 41-45.

Programa de educación musical, segundo año medio, Revista de Educación $\mathrm{N}^{\circ} 14$ (nueva época), número extraordinario con los programas de estudio del segundo año de enseñanza media (marzo, 1969), pp. 57-60.

Programa de educación musical, tercer año medio, Revista de Educación $\mathrm{N}^{\circ} 22$ (nueva época) (noviembre, 1969), pp. 55-58.

Programa de educación musical, cuarto año medio, Revista de Educación $\mathrm{N}^{\circ} 31$ (nueva época), programas del cuarto año de la enseñanza media de la rama científico-humanista (octubre, 1970), pp. 59-65.

REFORMA 1981

Planes y programas de estudio para la educación general básica. Educación musical, Revista de Educación No 79 (segunda edición, mayo 1980), pp. 102-106.

Educación musical, $1^{\circ}$ y $2^{\circ}$ año de la educación media, Revista de Educación, planes y programas de estudio de la educación media. Edición especial (marzo, 1982), pp. 76-79.

Educación musical, plan común: $1^{\circ}$ y $2^{\circ}$ año de Educación Media, Revista de Educación, $\mathrm{N}^{\circ}$ 94, planes y programas de estudio de la educación media (julio, 1985), pp. 73-77.

Educación musical, plan común: $3^{\circ}$ y $4^{\circ}$ año de Educación Media, Revista de Educación, $\mathrm{N}^{\circ}$ 94, planes y programas de estudio de la educación media (julio, 1985), pp. 164-169.

Decreto $\mathrm{N}^{\circ} 11201$, diciembre 18 de 1967, que fija planes de estudio de la escuela media. En Revista de Educación (nueva época), № 4 (marzo, 1968), Santiago: Publicación oficial del Ministerio de Educación Pública, pp. 17-22.

Ministerio de Educación (1965): Planes y programas para la Educación Básica y Media. (1981): Planes y programas para la Educación Básica y Media.

(1996): Objetivos Fundamentales y Contenidos Mínimos Obligatorios para la Educación Básica. Edición revisada de 2002.

(1998): Objetivos Fundamentales y Contenidos Mínimos Obligatorios para la Educación Media. Edición revisada de 2004.

Revista de EdUCACIÓN

$\mathrm{N}^{\circ} 5$ (abril, 1968); $\mathrm{N}^{\circ} 12$ (agosto, 1969) (segunda edición); $\mathrm{N}^{\circ} 14$ (marzo, 1969); $\mathrm{N}^{\circ} 22$ (noviembre, 1969); $\mathrm{N}^{\circ} 31$ (octubre, 1970); $\mathrm{N}^{\circ} 52 ; \mathrm{N}^{\circ} 53 ; \mathrm{N}^{\circ} 54$ (junio, 1975); $\mathrm{N}^{\circ} 63$ (julio-agosto, 1977); $\mathrm{N}^{\circ} 77$ (febrero-marzo, 1980); $\mathrm{N}^{\circ} 79$ (mayo, 1980); Nº 80 (junio-julio, 1980); N 87 (junio, 1981); Edición Especial (marzo, 1982); N 94 (julio, 1985). 